\title{
Modélisation numérique des nuages convectifs. Etude d'une ligne de grains intertropicale
}

\section{Constructing mathematical models of convective clouds. Study of an intertropical squall line}

\author{
J.P. Chalon et J.P. Lafore \\ Direction de la Météorologie \\ Centre National de Recherches Météorologiques, Toulouse
}

Une ligne de grains est composée de puissants cumulonimbus organisés en ligne et associés à de fortes averses, des orages et des coups de vent violents. Les lignes de grains des régions intertropicales présentent un intérêt primordial en Météorologie parce qu'elles foumissent la majeure partie des pluies des régions sahéliennes mais aussi, parce que participant aux transports énergétiques entre les régions équatoriales et les régions tempérées, elles apportent une forte contribution aux variations du temps observées à nos altitudes.

La connaissance de la structure de ces systèmes et de leur comportement a été jusqu'ici limitée par l'insuffisance des moyens d'observation à petite échelle, disponibles dans les régions sahéliennes.

\section{Objectifs scientifiques et campagne de mesure}

Une expérience d'étude de la Convection Profonde Tropicale (COPT) mettant en œuvre les moyens d'observation appropriés a été organisée avec la collaboration de nombreux laboratoires de recherche français et ivoiriens.

Les principales motivations de l'expérience COPT étaient les suivantes:

1) Améliorer la prévision d'occurence et d'intensité des évènements convectifs diurnes et des lignes de grains sur l'Afrique continentale,

2) Fournir des informations permettant de mieux décrire la structure des systèmes convectifs et leur évolution,

3) Parvenir à une amélioration des techniques de paramétrisation de la convection et des précipitations dans les modèles de prévision dynamiques et climatiques, 4) Analyser les effets électriques des orages de convection profonde, au sol et en altitude, pour améliorer la protection des bâtiments et des avions.

L'expérience principale s'est déroulée à Korhogo (Côte d'Ivoire) en mai-juin 1981. Le réseau d'observation étant composé (Fig. 1):
- de 2 radars Doppler,

- d'une station météorologique centrale avec station de réception des données satellite,

- d'une station de radiosondage,

- d'un réseau de stations météorologiques automatiques,

- d'un sondeur acoustique,

- d'un réseau de stations de mesure du champ élèctrique,

- d'une station de sondage en altitude du champ électrique.

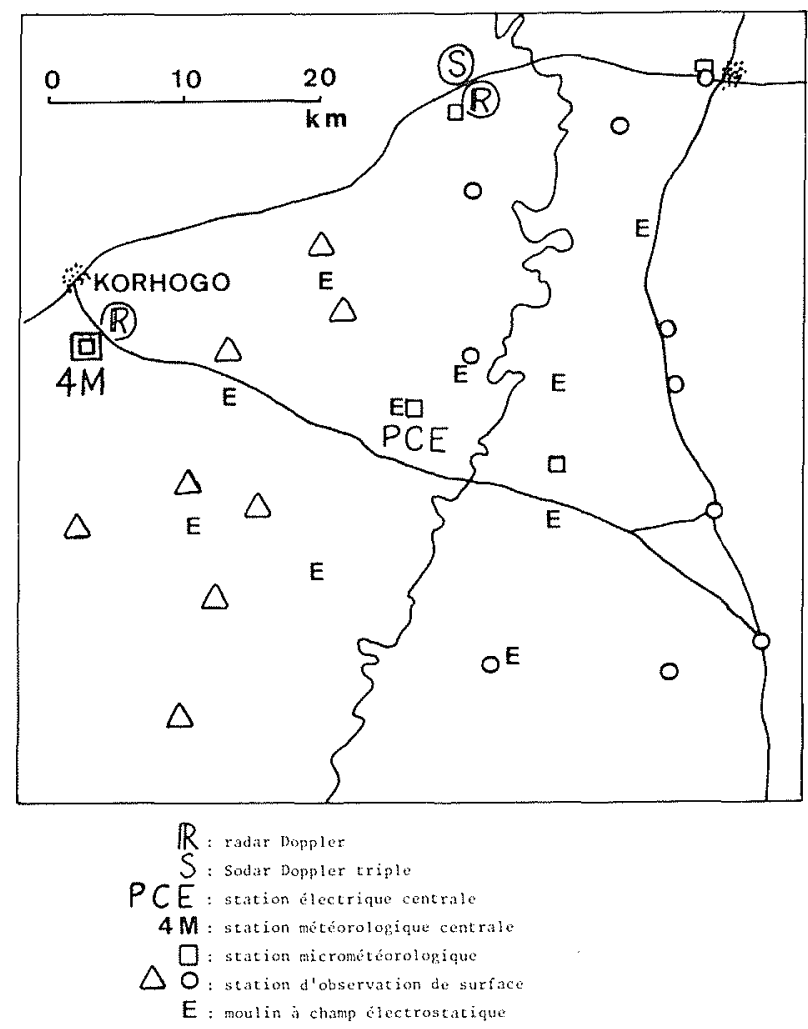

Figure 1 - Réseau instrumental utilisé pendant l'expérience COPT 81 (d'après COPT Organizing Commitee - 1982). 


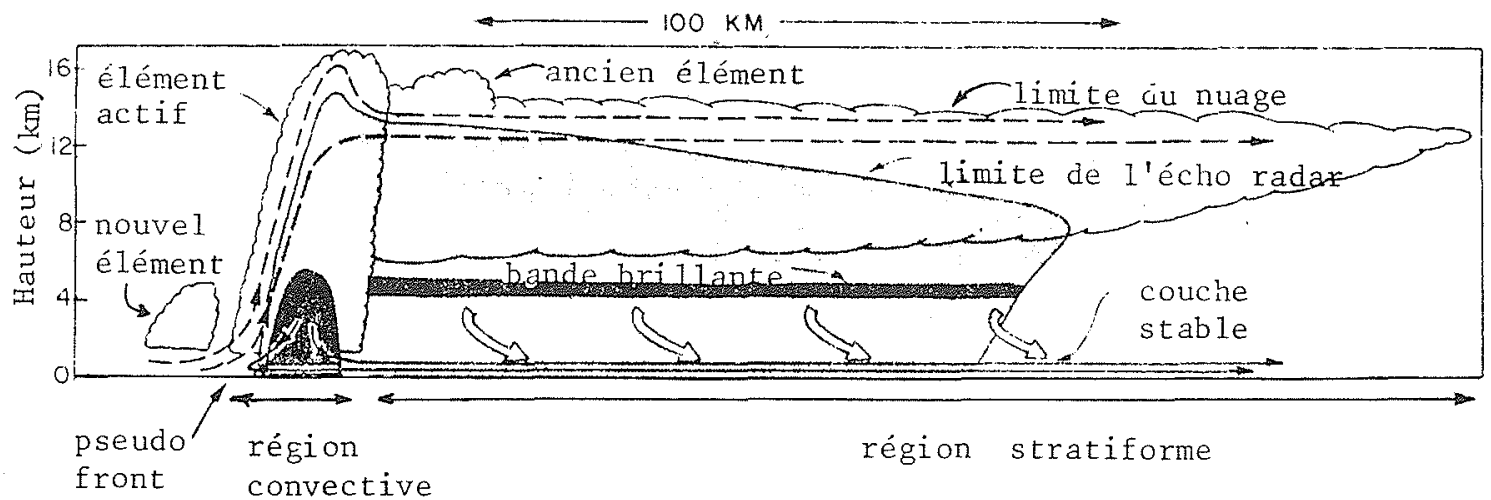

Figure 2 - Section droite schématique d'une ligne de grains. Les lignes de courant montrent les flux relatifs à la ligne. Les lignes tiretées représentent la circulation ascendante, les lignes continues, la circulation subsidente d'échelle convective et les flèches épaisses la circulation subsidente de mésoéchelle qui prend naissance sous la base des nuages dans la partie stratiforme. Les zones sombres indiquent les fortes réflectivités radar obtenues dans la bande brillante et dans la région de fortes précipitations associées à la région convective : les. zones grisėes, des régions de plus faible réflectivité radar, et les lignes ondulées, les contours extérieurs visibles đu nuage. (D'après Houze - 1977).

Les organismes participant à l'expérience étaient : - Le Centre National d'Etudes des Télécommunications (CNET)

- Le Centre de Recherches en Physique de l'Environnement (CRPE/CNET)

- L'Etablissement d'Etudes et de Recherches Météorologiques (EERM, Direction de la Météorologie)

- L'Institut et Observatoire de Physique du Globe de Clermont-Ferrand (IOPG)

- Le Laboratoire de Météorologie Dynamique (LMD)

- Le Laboratoire d'Aérologie (LA)

- L'Office National d'Etudes et de Recherches Aérospatiales (ONERA)

- L'Université d'Abidjan.

avec la collaboration :

- de l'Agence pour la Sécurité de la Navigation Aérienne (ASECNA)

et le support financier et logistique :

- de l'Institut National d'Astronomie et de Géophysique (INAG),

- de la "Direction des Recherches, Etudes et Techniques" (DRET)

- et du Gouvernement Ivoirien.

Le programme détaillé de cette expérience a fait l'objet d'une publication (COPT Organizing Committee1982).

\section{Description des lignes de grain et des condi- tions météorologiques associées}

\subsection{Les lignes de grain}

Les lignes de grain observées en Afrique Occidentale se déplacent généralement vers l'Ouest ou le Sud-Ouest à des vitesses d'environ 40 à $60 \mathrm{~km} / \mathrm{h}$ et s'étendent dans le sens Nord-Sud sur plusieurs centaines de kilomètres. Elles comprennent une partie frontale très active (région convective) suivie d'une enclume moins active (région stratiforme) pouvant s'étendre sur 150 à $200 \mathrm{~km}$ à l'arrière du système (Fig. 2). A petite échelle, une ligne est constituée de cellules convectives (cumulo-nimbus) d'une dizaine de kilomètres de diamètre qui pénètrent la tropopause et ont leur sommet à 16 ou $17 \mathrm{~km}$ d'altitude. Les nouvelles cellules de précipitation se forment à l'avant du système tandis que les plus anciennes se dissipent vers l'arrière où elles constituent une traine stratiforme dont le sommet dépasse rarement $14 \mathrm{~km}$ d'altitude.

Dans la partie avant, on observe des vitesses verticales ascendantes d'environ 10 à $20 \mathrm{~m} / \mathrm{s}$. A l'arrière, les vitesses verticales sont surtout subsidentes. Ces subsidences sont formées par de l'air qui est entrainé et refroidi par les précipitations, se rapproche du sol et s'étale dans les basses couches. Au niveau du front avant de la ligne, cet air froid renforce la convergence et le contraste thermique avec le flux de mousson. Ceci a pour effet de renforcer la convection et d'en assurer le maintien. Les lignes de grains peuvent ainsi se déplacer à des vitesses supérieures à celles des vents observés à tous les niveaux et se maintenir même dans des conditions peu favorables aux développements convectifs. Ceci leur permet de poursuivre leur activité la nuit et au-dessus de régions peu propices à la convection. Une ligne parvient ainsi à traverser et à arroser des régions de plusieurs centaines de milliers de kilomètres carrés.

\subsection{La structure de l'atmosphère et son évolution}

En Afrique Occidentale, l'atmosphère dans laquelle se développent les lignes de grain comporte, dans les 1000 premiers mètres au-dessus du sol un flux de mousson chaud et humide venant du Sud ou du Sud Ouest, surmonté jusqu'à environ $15 \mathrm{~km}$ par une couche d'air sec venant de l'Est et présentant souvent un maximum de vitesse vers $3 \mathrm{~km}$ d'altitude et un autre vers $12 \mathrm{~km}$.

Le réchauffement solaire, l'évapotranspiration des sols, l'apport d'humidité par le flux de mousson augmentent la quantité d'énergie disponible sous formes sensible et latente dans les basses couches, créant ainsi un fort déséquilibre de la répartition verticale de l'énergie 
dans la troposphère (Fig. 3). Ceci a pour effet d'augmenter l'instabilité convective et de favoriser la formation de nuages convectifs ou le passage de lignes de grains.

Lors de son passage, la ligne de grain va réduire ou supprimer ce déséquilibre en redistribuant l'énergie (humidification et réchauffement de l'air situé dans la moyenne troposphère, refroidissement et asséchement de l'air dans les basses couches). La ligne de grain se comporte comme une machine thermique et joue dans l'atmosphère un rôle stabilisateur.

\subsection{Précipitations}

Les lignes de grain fournissent la majeure partie des précipitations qui concernent les régions sahéliennes. Une seule ligne peut arroser des régions de 5 ou $600000 \mathrm{~km}^{2}$.

La partie convective est associée à des précipitations très actives (de l'ordre de $100 \mathrm{~mm} / \mathrm{h}$ ), la partie stratiforme moins active est associée à des pluies faibles ou modérées (quelques $\mathrm{mm} / \mathrm{h}$ ) mais qui sont plus étendues et peuvent donner lieu à des quantités totales de précipitations aussi importantes que celles enregistrées sous la partie convective.

Par exemple, pour une ligne de grain d'intensité moyenne, qui est restée active pendant 23 heures, Houze (1977) a évalué une quantité totale de précipitations d'environ $4,6 \times 10^{12} \mathrm{~kg}$ dont $40 \%$ provenaient de la partie stratiforme à l'arrière du système.

\section{Présentation du modèle}

La modélisation numérique permet de simuler l'évolution de différents paramètres dynamiques et thermodynamiques en chaque point d'une grille régulière couvrant un domaine à l'intérieur duquel se produit le phénomène étudié.

Le modèle de convection profonde présenté ici couvre actuellement un domaine parallélipipédique de $30 \mathrm{~km}$ d'extension horizontale et de $20 \mathrm{~km}$ d'épaisseur dans le cas d'une maille élémentaire de $1.5 \mathrm{~km}$ horizontalement et $800 \mathrm{~m}$ verticalement.

Il calcule l'évolution de 9 variables :

- les trois composantes de la vitesse de l'air, la pression, la température potentielle, le rapport de mélange en vapeur d'eau, les rapports de mélange en eau liquide nuageuse et en eau liquide précipitante enfin une énergie turbulente à l'échelle inférieure à la maille.

Le système d'équations (LAFORE 83) gérant les évolutions de ces paramètres est formé des équations de base de la météorologie. Soient :

- une équation d'état,

- les équations de la dynamique dérivées de NavierStockes,

- l'équation de continuité,

- une équation thermodynamique pour la température potentielle.

Pour le cycle de l'eau et des précipitations la paramétrisation utilisée (du type KESSLER 69) prend en compte :

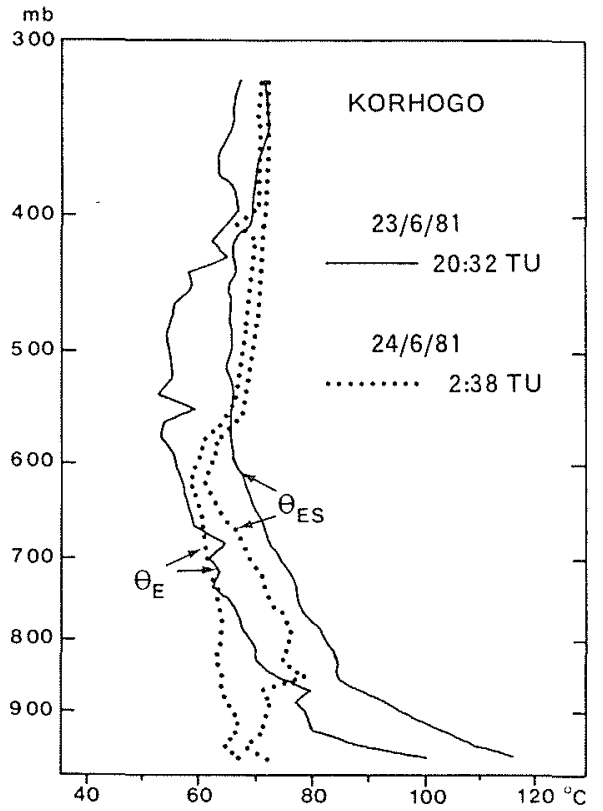

Figure 3 - Profils verticaux de température potentielle équivalente $\theta_{E}$ et de température potentielle équivalente saturée $\theta_{E S}$ observées avant (traits pleins) et après (traits pointillés) le passage de la ligne de grains des 23-24 juin 1981 sur le site de Korhogo. Ces profils mettent en évidence une forte instabilité de l'atmosphère précédant le passage de la ligne. Derrière, le profil est plus stable, l'atmosphère a été stabilisée sur une grande épaisseur.

- la production de gouttes de pluie à partir des gouttelettes de nuage par auto-conversion et accrétion,

- l'évaporation des gouttes de pluie

- et leur chute par rapport à l'air ambiant.

La phase solide (glace) du cycle de l'eau n'est pas (actuellement) prise en compte.

Une équation d'évolution pour l'énergie cinétique turbulente permet de représenter les phénomènes turbulents d'échelle inférieure à la maille. Le modèle tient compte également de la condensation à l'échelle inférieure à la maille.

Le domaine de simulation est très réduit comparativement à la taille des lignes de grains étudiées. l'introduction de conditions limites latérales (CLL) mixtes a permis de simuler la partie avant de la ligne où l'activité convective est maximale.

Ces CLL mixtes supposent une périodicité le long de la ligne convective à l'avant et à l'arrière du domaine de simulation, les évolutions des différents paramètres sont déterminées en fonction de l'état de la convection à l'intérieur du domaine et du forcing des échelles supérieures.

\section{Description d'une simulation sur la ligne de grain du 23/24 juin 1981}

Les mesures ne fournissent pas actuellement une description suffisamment détaillée de l'état de l'atmosphère associée aux phénomènes convectifs pour permettre une initialisation d'un modele tridimensionnel. Dans notre cas la convection est déclenchée par 

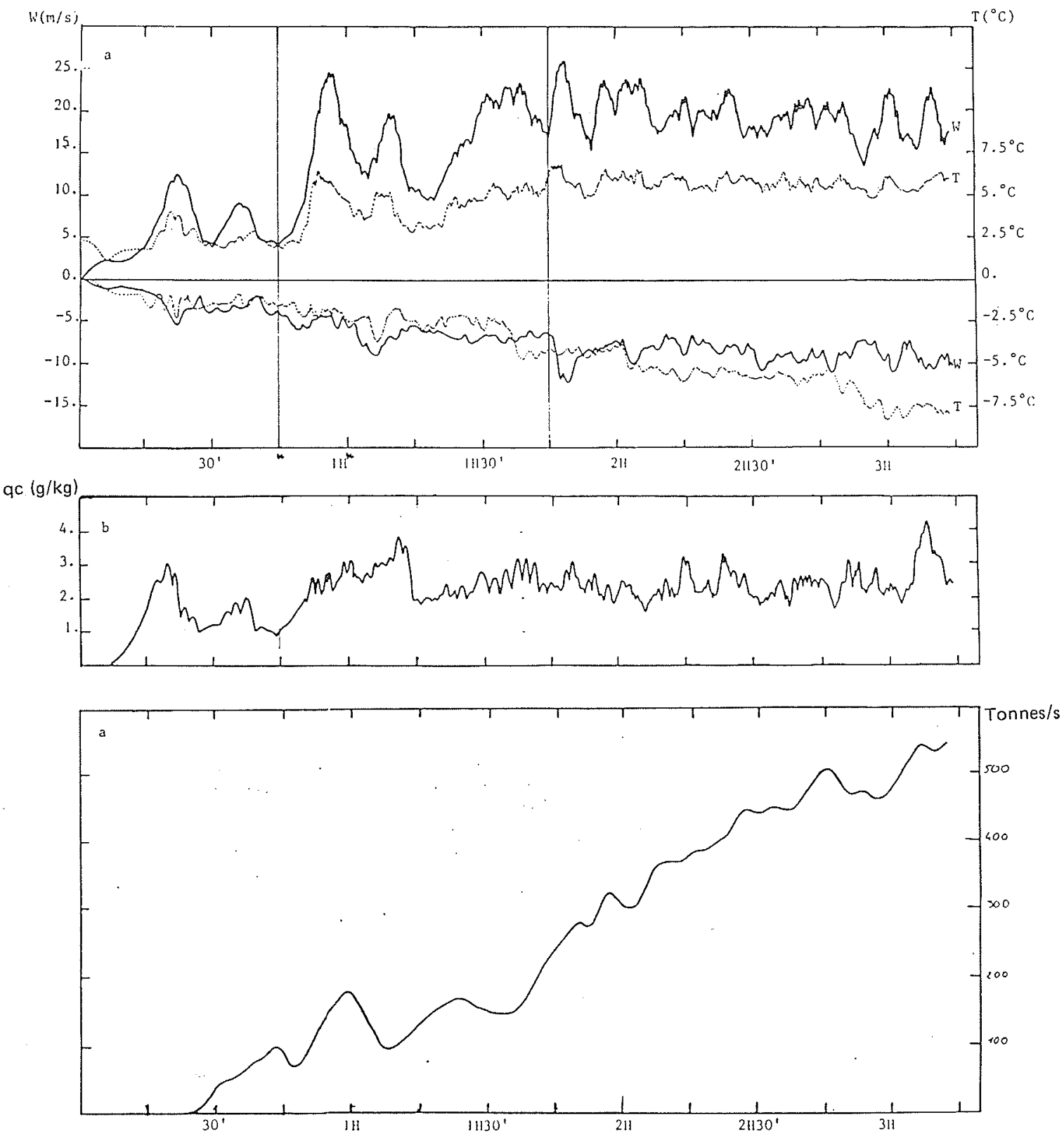

Figure $4 \mathrm{a} b c-$ Simulation de la ligne de grains du 23-24 juin 1981 (COPT 81):

a) Evolution des maxima et minima de vitesses verticales $W$ et d'écarts de température $T$

b) Du maximum d'eau de nuage $q_{c}$

c) Du flux de précipitation au sol

l'introduction d'une perturbation de la température dans une atmosphère horizontalement homogène, caractéristique de l'environnement précédant le passage de la ligne de grains. Dans le cas de la situation du $23 / 24$ juin 1981 , nous avons choisi le radiosondage de $20 \mathrm{~h} 32$ (Fig. 3) effectué $3 \mathrm{~h}$ avant le passage de la ligne.

Le déroulement de la simulation d'une durée de $3 \mathrm{~h} 15$ se décompose en trois phases (Fig. 4):

\section{1) De 0 à $45 \mathrm{mn}$ : l'initialisation}

Une bulle chaude bidimensionnelle permet d'initialiser la convection dans cette phase. L'écoulement reste donc bidimensionnel. Lorsque les premiers noyaux de précipitation atteignent le sol, de nouvelles cellules convectives se développent à l'avant du système. La ligne se déplace à une vitesse d'environ $5 \mathrm{~m} / \mathrm{s}$ et les vitesses verticales maximales sont de l'ordre de $10 \mathrm{~m} / \mathrm{s}$.

\section{2) de $45 \mathrm{mn}$ à 1 h $45^{\prime}$ : la phase transitoire}

Nous introduisons de façon aléatoire quelques noyaux de précipitation pour rompre la structure bidimensionnelle de l'écoulement qui devient ainsi tridimensionnel avec apparition de plusieurs cellules d'intensité plus forte $(25 \mathrm{~m} / \mathrm{s})$. On obtient un régime transitoire pendant lequel l'intensité convective de la ligne et sa vitesse de propagation augmentent. 


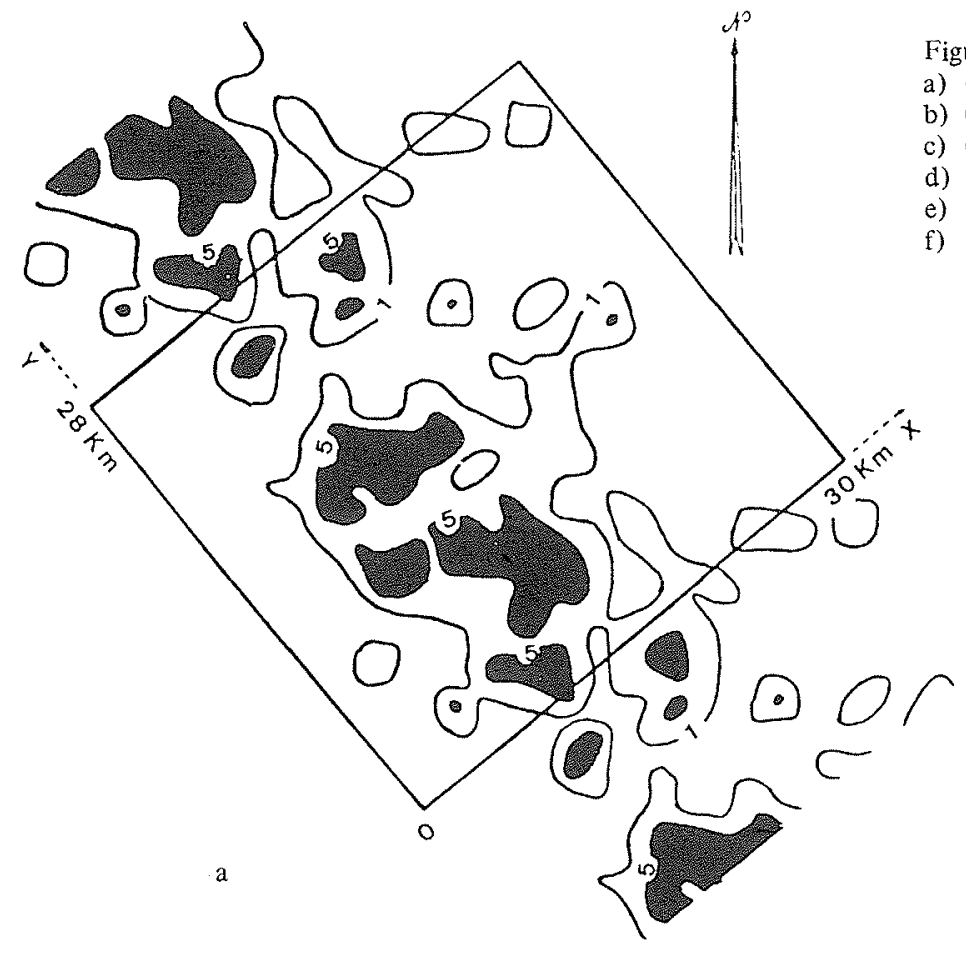

Figure $5 \mathrm{a} \mathrm{b} \mathrm{c}$ d e f - Résultats obtenus après $2 \mathrm{~h} 30$ de simulation.

a) Coupe horizontale d'eau de nuage $q_{c}(\mathrm{~g} / \mathrm{kg})$ à l'altitude $Z=3,6 \mathrm{~km}$

b) Coupe verticale des vitesses verticales $W(\mathrm{~m} / \mathrm{s})$

c) Coupe verticale des vitesses horizontales $U(\mathrm{~m} / \mathrm{s})$

d) Coupe verticale de l'eau de nuage $q_{c}(\mathrm{~g} / \mathrm{kg})$

e) Coupe verticale des écarts de température potentielle $\theta\left({ }^{\circ} \mathrm{C}\right)$

f) Coupe verticale de l'eau de pluie $q_{r}(\mathrm{~g} / \mathrm{kg})$
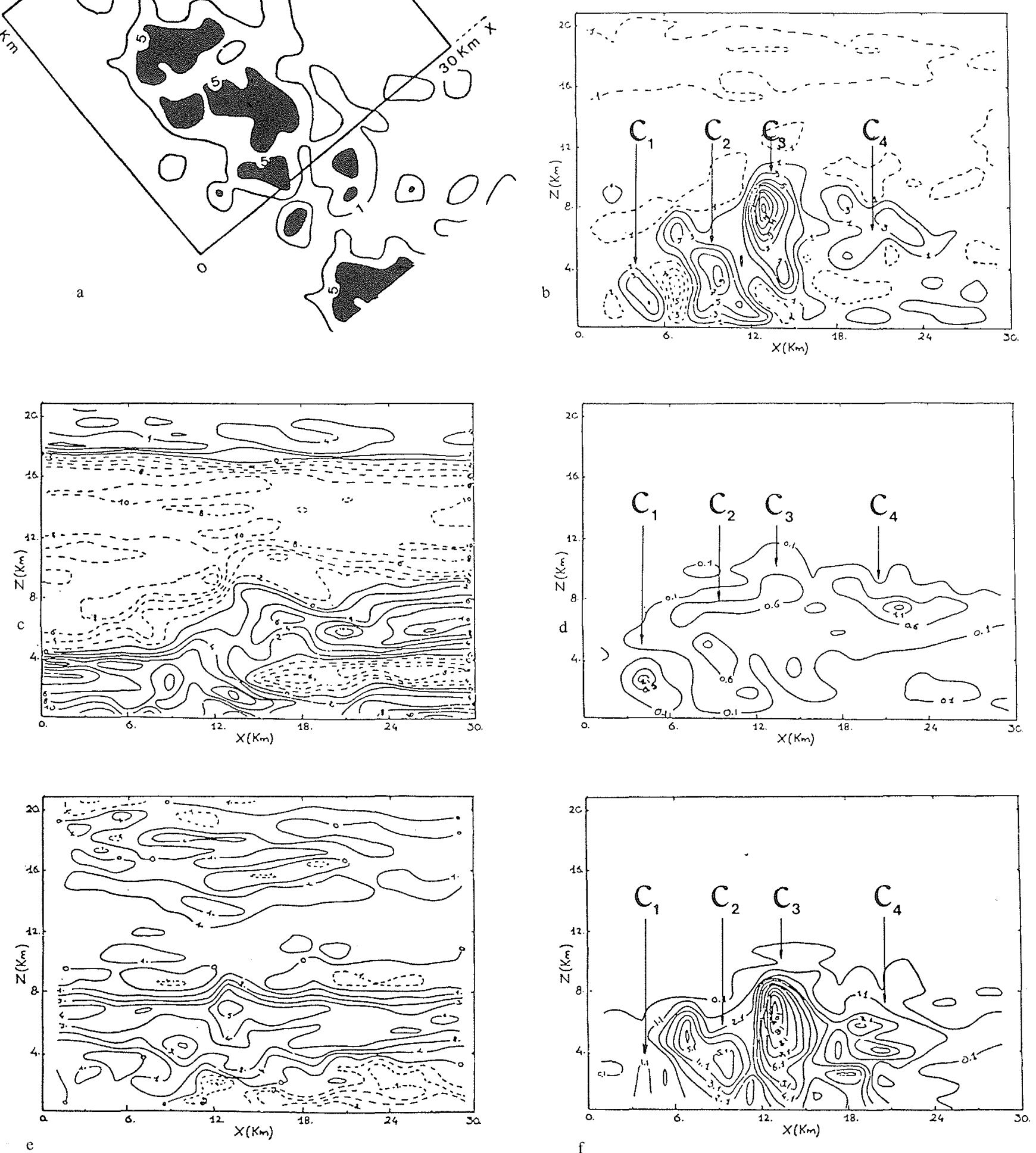

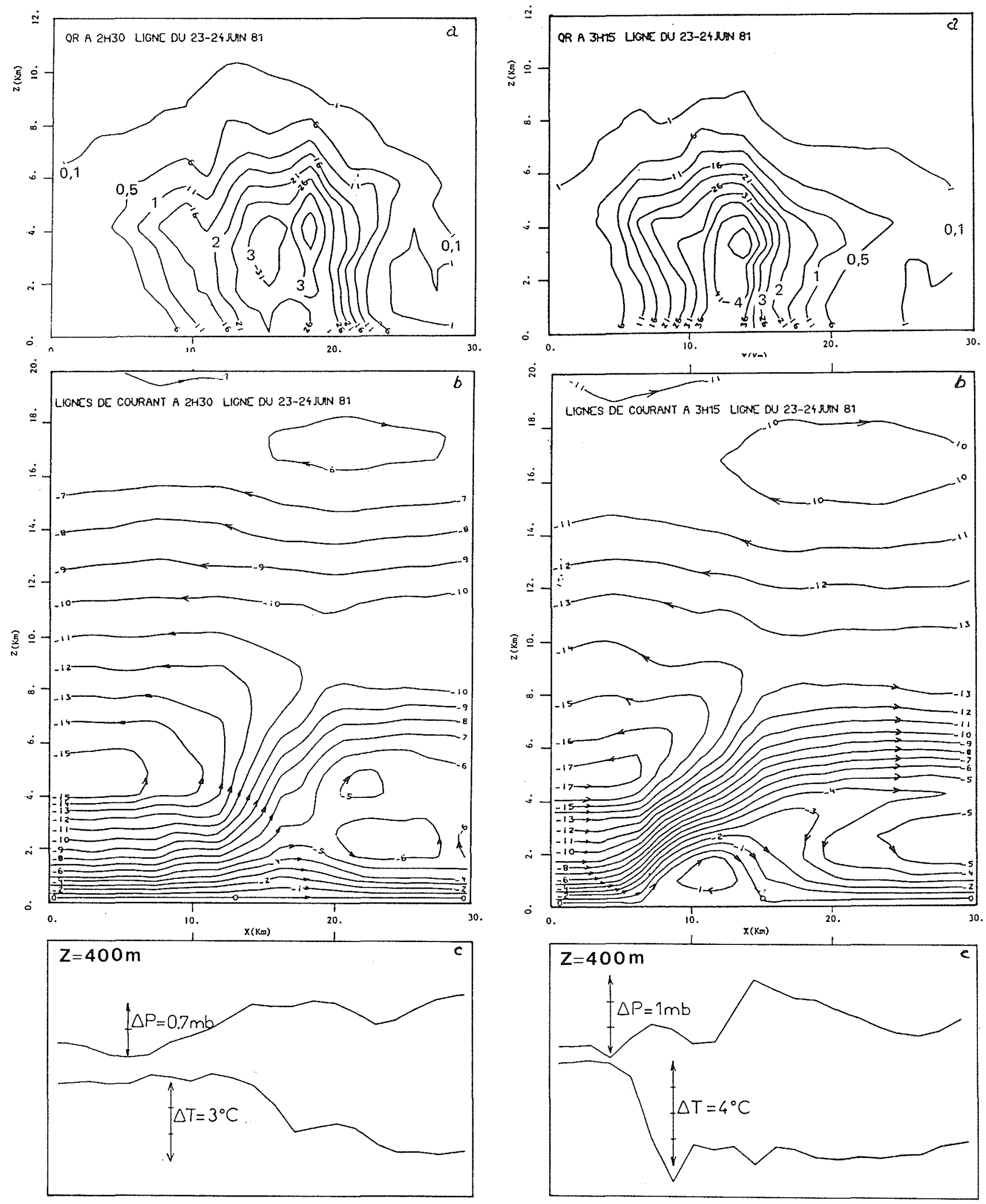

Figure $6 \mathrm{ab} c$ - Image bidimensionnelle moyenne de la ligne du $23 / 24$ juin COPT 81 dans le plan vertical perpendiculaire à sa direction après $2 \mathrm{~h} 30$ de simulation:

a) pour l'eau précipitante $\left(\mathrm{g} / \mathrm{m}^{3}\right)$

b) pour des lignes de courant $\left(\rho_{0} U\right)$ dans le repère lié au déplacement de la ligne convective $(-9 \mathrm{~m} / \mathrm{s})$

c) Evolution de la pression et de la température moyenne au niveau $400 \mathrm{~m}$ dans la direction perpendiculaire à la ligne.

Figure $7 \mathrm{a}$ b c - Comme précédente (Fig. 6) avec une vitesse de translation de $-10.2 \mathrm{~m} / \mathrm{s}$ après 3 h 30 de simulation. 


\section{3) Phase d'étude}

Au bout de $1 \mathrm{~h} 45^{\prime}$ de simulation le système atteint un régime permanent et commence à s'autoentretenir. Ses caractéristiques se rapprochent peu à peu de celles de la ligne de grains qui a été observée.

\subsection{Description morphologique}

La coupe horizontale d'eau de nuage (Fig. 5a) donne une vue d'ensemble de la morphologie de la ligne. On distingue la taille des noyaux les plus actifs et les nouvelles cellules à l'avant du système. Des séries de coupes verticales permettent de décrire la structure du système (Fig. 5). On distingue ainsi une succession de cellules à des phases différentes de leur cycle de vie, soient :

$-C_{1}$ une nouvelle cellule qui se développe à l'avant du système,

- $\mathrm{C}_{2}$ une cellule qui commence à produire des précipitations,

- $\mathrm{C}_{3}$ une cellule au maximum de son activité produisant un fort noyau de précipitation,

- $\mathrm{C}_{4}$ une cellule en voie de dissipation qui s'étale à l'arrière du système.

\subsection{Circulation moyenne perpendiculaire à la ligne}

Pour mieux analyser les résultats, les champs simulés sont utilisés pour composer une image bidimensionnelle moyenne de la ligne dans un plan vertical perpendiculaire à celle-ci.

Les figures 6 et 7 donnent ainsi une image de l'écoulement moyen à l'intérieur de la ligne.

L'air humide et instable du flux de mousson alimente par l'avant du système un fort courant ascendant $(6 \mathrm{~m} / \mathrm{s}$ en moyenne) qui se "divise en deux". Une partie s'évacue vers l'arrière en réchauffant les niveaux moyens et supérieurs ; une autre partie entraînée par les précipitations redescend et s'étale près du sol en refroidissant les basses couches par évaporation. Les courants descendants sont plus forts $(-2 \mathrm{~m} / \mathrm{s})$ dans la partie avant de cette masse d'air tandis qu'ils sont plus faibles $(-0,5 \mathrm{~m} / \mathrm{s})$ à l'arrière de la ligne. L'air froid, chargé de précipitations, favorise à son tour le soulèvement du flux de mousson en formant un obstacle qui s'oppose à son avance.
On retrouve ainsi le mécanisme principal d'évolution des lignes de grains avec l'entretien du contraste thermique par les subsidences et la formation de nouvelles cellules à l'avant du système. La circulation à 3 h 15 est du même type que celle observée à 2 h 30 mais est plus intense.

Cette ligne simulée est associée (Fig. $6 \mathrm{c}$ ) à un saut de pression (de l'ordre de $1 \mathrm{mb}$ ) et à une chute de température (de 2 à $4^{\circ} \mathrm{C}$ ) qui correspondent sensiblement aux valeurs qui ont été observées au sol lors de l'expérience.

Ces discontinuités permettent de localiser avec précision le front de rafale et de mesurer la vitesse de propagation du système.

On constate une accélération de la ligne avec des vitesses de propagation de $9 \mathrm{~m} / \mathrm{s}$ après $2 \mathrm{~h} 30$ de simulation et de $10.2 \mathrm{~m} / \mathrm{s}$ après $3 \mathrm{~h} 15$. La vitesse estimée à partir des observations (réseau sol et images radar) fluctue entre 11 et $13 \mathrm{~m} / \mathrm{s}$. La figure $4 \mathrm{c}$ montre une augmentation constante des précipitations atteignant le sol sur tout le domaine. La valeur obtenue de 600 tonnes/s à la fin de la simulation est nettement inférieure à l'estimation de 3000 tonnes/s effectuée d'après les mesures de pluviométrie.

\subsection{Conclusion}

Ce modèle est un outil performant pour étudier les lignes convectives. La comparaison avec les données recueillies sur une situation observée montre qu'il reproduit les caractéristiques générales de la ligne de grains, la circulation générale et les modifications dynamiques et thermodynamiques de l'atmosphère à l'arrière du système.

Le domaine de simulation est limité par la taille du calculateur utilisé. Avec un domaine restreint actuellement à $30 \mathrm{~km}$ le modèle est incapable de reproduire la zone stratiforme à l'arrière de la ligne. Cette zone joue un rôle important dans la dynamique du système, ce qui peut expliquer la trop faible activité convective des nuages simulés. Nous envisageons actuellement d'augmenter la taille du domaine simulé en utilisant un ordinateur plus performant (CRAY 1).

L'étude présentée a été entreprise dans le cadre de l'expérience COPT 81 et sera appliquée par la suite aux systèmes frontaux convectifs qui seront étudiés en France lors de l'expérience FRONTS 84.

\section{Bibliographie}

C.O.P.T. Organizing Comittee. - "C.O.P.T. 81 - a field experiment designed for the study of dynamics and electrical activity of deep convection in continental Tropical regions." Conference on Cloud Physics. A.M.S. Chicago p. 580, 1982.

HOUZE, R.A. - "Structure and dynamics of a tropical Squall line system". M.W.R. 105 p. 1540, 1977.

KESSLER E. - "On the distribution and continuity of water substance in atmospheric circulation." Meteor. Monog. n 32 Amer. Meteor. Soc., 1969.

LAFORE J.P. - Sensitivity of a Cloud Model to the Lateral Boundary Conditions and Subgrid-Scale Parameterisation. Conference on Cloud Physics. Chicago p. 524-526, 1982.

LAFORE J.P. - "Présentation d'un modele $3 \mathrm{D}$ de convection profonde." Note E.E.R.M. à paraître, 1983. 


\section{Discussion}

Président : R. HLAVEK

Le Président. - Nous terminons sur un sujet qui, sauf erreur de ma part, n'est pas uniquement le radar à précipitations dont on a déjà parlé à plusieurs reprises. Le travail a été fait en Côte d'Ivoire, dans la région de Khorogo. Il s'agit là d'un problème très important pour l'Afrique sahélienne : les systèmes convectifs profonds tropicaux ont une grande importance au point de vue de la vie dans ces régions.

M. LAFORE. - Je remercie M. TARDIEU qui a montré quelques photographies de radar d'une des situations qui nous concerne, l'expérience COPT 81.

Le Président. - Nous allons céder la parole aux assistants.

M. WALDVOGEL. - Pouvez-vous développer la comparaison avec les autres modèles similaires?

M. LAFORE. - 11 existe des modèles d'échelle supérieure, mais qui ont une résolution maximum d'une dizaine de kilomètres et même plus proche de la limite de Péridot. Mais il n'y a pas de simulation d'une ligne de grains de ce type. Nous utilisons une échelle de 35 kilomètres. Nous travaillons avec le LMD (Laboratoire de Météorologie Dynamique). Ils n'ont pas fait encore de simulation, mais ils vont utiliser ces premiers résultats.

Le Laboratoire de Clermont a un modèle de philosophie analogue à la nôtre. Pour l'instant, je ne sais pas où ils en sont ; mais c'est un modèle d'échelle un peu supérieure et, à l'intérieur de ce modèle, on paramétrise la convection à l'aide d'un modèle à symétrie axiale, modèle unidimensionnel avec plusieurs tubes imbriqués. Ce pourrait être en effet un modèle complémentaire. Mais il n'admet pas l'hypothèse de périodicité; il traite tous les 1000 kilomètres ensemble, et vu les tailles d'ordinateurs qu'il y a actuellement cela me paraît impossible. Nous avons décrit une limite à la partie avant et pas du tout le retour arrière. Pour la dynamique, à mon avis, les clés de la ligne de grains sont dans la présence, à l'arrière de la ligne de grains, d'une vaste zone froide qui s'écoule lentement sur le sol et forme une sorte de charrue, un soc qui soulève devant lui une atmosphère qui est plus ou moins instable. Cette activité peut crô̂tre et décrôtre et il y a une permanence; si bien que, grâce à ce soulèvement régulier, il y a une zone de précipitations de méso-échelle, et la ligne de grains continue à se propager malgré le refroidissement nocturne. Sur une photo satellite, pour la convection pure, les petits points disparaissent; par contre, restent ces grandes lignes continues. Les lignes de grains ont une tendance à perdre leur activité la nuit, mais elles parviennent à survivre.

M. $A M A Y E N C$. - Je voudrais signaler les travaux de MONCRIEFF et MILLER, en bidimensionnel. Ils partent du principe que les lignes de grains ont une symétrie dans la zone supposée homogène dans la direction perpendiculaire au déplacement. Ils aboutissent à des résultats qui sont tout à fait simi- laires à ceux qui viennent d'être montrés.

C'est très intéressant parce qu'il n'y a pas d'hypothèse de périodicité ; c'est plus satisfaisant que de représenter l'extension de la ligne de grains par des interactions aux limites du domaine du modèle.

M. LAFORE. - La simulation de MILlER a une particularité : ils obtiennent une sensibilité des résultats énorme au type de profil de vent et, pour moi, c'est lié uniquement au modèle. Trouver une structure stationnaire dans un cas bidimensionnel, cela peut arriver à la limite, mais c'est exceptionnel ; alors que, dans la nature, quand on voit des lignes de grains qui passent c'est vraiment impressionnant : on voit des séquences de profil de vent sur toute la surface et on voit le passage des lignes; à certains moments, par périodes, il y en a trois pendant trois jours, cela n'arrête pas; l'atmosphère se renouvelle tout de suite, l'instabilité, le flux. .

Il est donc très utile de travailler en parallèle. Ce qu'on voudrait parvenir à faire petit à petit, c'est développer un outil tridimensionnel, mais comparer toujours à des structures bidimensionnelles moyennées et voir ce que la troisième dimension apporte. Le bidimensionnel permet aussi de développer des théories semi-analytiques pour comprendre ce type d'écoulement.

Trouver une méthodologie dans ce domaine, je crois que c'est actuellement très important. Il y a de très bons modèles, entre autres aux Etats-Unis, orientés principalement vers la morphologie ; mais quel est le bilan quantitatif ?

Le Président. - Je pense que le modèle bidimensionnel anglais peut "trouver" des cellules à l'avant des lignes de grains ?

M. LAFORE. - Ils ont des cellules, mais on n'a aucune idée de la variance de leurs résuitats. Ils calculent des écoulements moyens. Nous sommes en train de changer le modèle des simulations que j'ai faites avec Vincent BETCHER cette année; nous allons faire les mêmes sur le modèle LMD avec des modifications. L'avantage de leur modèle est qu'on peut le transformer en bidimensionnel. Si bien qu'on a plusieurs modèles pour certaines expériences : si l'écoulement stationnaire produit par le modèle tridimensionnel arrive à se maintenir, l'écoulement peut devenir bidimensionnel.

D'autre part, actuellement, l'équipe du CRPE qui travaille sur les données de radar DOPPLER analyse deux reconstitutions de situations et on s'aperçoit que ces deux situations sont entièrement différentes : une situation où, localement sur $50 \mathrm{~km}$, on a un écoulement bidimensionnel ; en réalité, quand on regarde à plus grande échelle, à méso-échelle, il peut y avoir un effet de tourbillons horizontaux; mais l'autre situation, celle que nous avons simulée, est beaucoup plus multicellulaire, c'est-à-dire qu'on a des celludes côte à côte. 
Abstract

Constructing mathematical models of convective clouds. Study of an intertropical squall line

Precipitation is the culmination of a long chain of physical processes. By means of a mathematical model the dynamics involved in the formation of precipitation can be stimulated and certain parameters not directly measurable can be evaluated.

In this article we describe the effect of precipitation on the maintenance of a convective system in the case of tropical convection: the squall line. The results from the model are considered in relation to measurement and, in particular, in relation to data obtained from DOPPLER radars. This study is now being carried out as part of the COPT 81 experiment and will subsequently be applied to frontal convective systems, which are particularly important for pluviometry in our temperate zones and will be examined in a coming measurement campaign - FRONT 84. 